\title{
What are the biological and therapeutic implications of biomolecule corona formation on the surface of inhaled nanomedicines?
}

\author{
"...we are only at the very beginning of a long journey to understand nano-bio \\ interactions in the lungs."
}

\begin{abstract}
Keywords: alveolar macrophages $\bullet$ complement $\bullet$ inhaled nanomedicines $\bullet$ protein corona - pulmonary drug delivery $\bullet$ respiratory tract lining fluid $\bullet$ surfactant proteins
\end{abstract}

\section{Respiratory tract lining fluid is not blood}

It may be a statement of the obvious, but one would not expect the corona formed on a nanoparticle surface following deposition in the lungs to resemble that surrounding nanoparticles after exposure to plasma or whole blood, or for that matter cell culture medium. This is self-evident; the only problem is, no one has quite shown it yet.

Respiratory tract lining fluid (RTLF) is compositionally very different from blood plasma; varying in ionic strength, antioxidant, lipid and protein content. RTLF composition also varies markedly throughout the length of the respiratory tract, reflecting local contributions from mucus-secreting cells in the major conducting airways and surfactant from type II pnuemoctyes in the alveolar region. While the RTLF does contain a wide variety of plasma-derived proteins including albumin, transferrin, apolipoprotein A-1, haptoglobin, $\alpha$-1-antitrypsin and $\alpha-2$-macroglobulin [1]; its proteome is also rich in lung-specific proteins, such as Clara cell secretory protein 16 and pulmonary surfactant proteins $A$ and $D$, which play important roles in innate host defense [2]. In addition, in the alveolar region, surfactant proteins $\mathrm{B}$ and $\mathrm{C}$ help to stabilize the monolayer of phospholipids (primarily phosphatidylcholine, phosphatidylglycerol) and cholesterol, which lowers the surface tension and prevents alveolar collapse during expiration [3]. It follows that the corona formed on the surface of an inhaled particle incident at the air-lung interface will reflect the local composition of the RTLF, and comprise a variety of different biomolecules such as phospholipids in addition to proteins. It is thus more accurate to speak of a 'biomolecule' corona rather than a 'protein' corona in this case.

In collaboration with several other groups, we have recently begun to characterize the biomolecule corona formed on the surface of silica nanoparticles when incubated within concentrated bronchoalveolar lavage fluid samples obtained from healthy humans and patient groups with various lung diseases. For this initial characterization, silica nanoparticles were chosen as a model particle system because their protein corona formation has been studied extensively in human plasma and fetal bovine serum. Mass spectrometry was used to characterize the protein component of the biomolecule corona, identifying over 400 distinct proteins on the particle surface following incubation in RTLF from healthy young adults [4]. In contrast, a significantly lower number of proteins adsorbed to silica nanoparticles incubated in human plasma, ranging from approximately 63 to 166 distinct proteins in different stud-

\begin{abstract}
Abhinav Kumar
Institute of Pharmaceutical Science, King's College London, London, UK

Ben Forbes

Institute of Pharmaceutical Science, King's College London, London, UK

Ian Mudway

MRC-PHE Centre for Environment \& Health, Analytical \& Environmental Sciences, King's College London, London, UK
\end{abstract}

\section{Elif Melis Bicer}

MRC-PHE Centre for Environment \& Health, Analytical \& Environmental Sciences, King's College London, London, UK

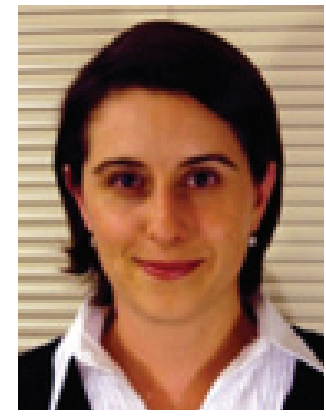

Lea Ann Dailey Author for correspondence: Institute of Pharmaceutical Science, King's College London, London, UK lea_ann.dailey@kcl.ac.uk 
ies [5,6]. Not surprisingly, the type of proteins involved in biomolecule corona formation in the RTLF differed considerably from that of the plasma. While the protein corona formed in plasma was rich in proteins involved in coagulation pathways (e.g., fibrinogen, kininogen, prothrombin) [5], the biomolecule corona formed following incubation in RTLF was significantly enriched with surfactant (e.g., SP-A, SP-B and SP-D) and complement (e.g., C1q, C2, C3, C4) proteins relative to their concentration in the RTLF [4]. Conceptually, it makes sense from a physiological perspective that the corona formed is enriched with proteins that promote particle clearance from the lungs.

\section{"The ideal properties of an inhaled nanomedicine are very different to many of the systems that have been used as models for inhaled nanomedicine research."}

\section{Inhaled nanomedicines: what materials should we be studying?}

The literature on the fate of different nanomaterials in the lungs has grown dramatically over recent years and has substantially improved our understanding of the relationship between particle physicochemistry and nanomaterial toxicity/biocompatibility. Many of these studies purport to generate data of direct relevance for the development of inhaled nanomedicines. However, when taking a closer look, there are still very few published studies which investigate lung-specific nano-bio interactions $[7,8]$ and lung biocompatibility [9-12] using nanomaterials that have been purposely developed as inhaled nanomedicines.

The ideal properties of an inhaled nanomedicine are very different to many of the systems that have been used as models for inhaled nanomedicine research. For example, inhaled nanomedicines should dissolve or biodegrade rapidly in the lungs to avoid accumulation upon multiple dosing [13], should be stealth-coated or hydrophilic [12] in nature as well as exhibit a relatively neutral or low-density surface charge to avoid immune activation in the lungs and finally, should interact minimally with components of the RTLF, so as to avoid perturbation of normal physiological processes of the lungs [14]. Interestingly, there is also some evidence that the rigidity of a nanomaterial may play a role in biocompatibility, with more rigid nanoparticles promoting enhanced macrophage responses compared with less-rigid, gel-like nanoparticles of the same material [15]. More studies that investigate drug delivery relevant materials such as nanosized drug particles, lipid-, protein- and biopolymer-based nanosystems, as well as suitable inorganic nanomaterials, for example, calcium phosphate, would do much to improve our understanding of the fate of different nanomedicines in the lungs.

\section{Biological \& therapeutic implications for inhaled nanomedicines}

What is the significance of biomolecule corona formation on the surface of inhaled nanomedicines? It is easy to envision that the nature of the corona will affect the dissolution or biodegradation rate of the nanomedicine and that particle recognition by scavenger cells, such as alveolar macrophages, may be enhanced or the clearance kinetics of the particulate material may be altered. For example, the adsorption of dipalmityl phosphatidylcholine has been shown to increase the wettability of hydrophobic drugs (hydrocortisone) to allow an enhanced dissolution rate [16]. It has also been established recently that protein and biomolecule corona formation may mask targeting moieties attached to the particle surface, thereby reducing the potential for cell-specific targeting of nanomedicines [17]. Additionally, it has been demonstrated that SP-D coating of nanoparticles [18] and SP-A and C1q protein-protein interaction [19] promote particle phagocytosis. If more were understood about the relationship between the surface chemistry of relevant nanomedicine materials and biomolecule corona formation in the lungs, systems could be engineered to specifically avoid corona formation (e.g., stealth technologies) or attract specific proteins to achieve an in situ targeting effect [20], which could be used for therapeutic advantage. However, as highlighted above, we are only at the very beginning of a long journey to understand nano-bio interactions in the lungs. Meanwhile, the incidence of respiratory disease is increasing worldwide and a new generation of inhaled therapeutics is required urgently for this unmet need. Fundamental studies to characterize the lung-specific biomolecule corona formed on the surface of nanoparticles relevant for therapeutic use in the lungs are the first step in understanding and exploiting the biological and therapeutic significance of this phenomenon.

\section{Financial \& competing interests disclosure}

The authors have no relevant affiliations or financial involvement with any organization or entity with a financial interest in or financial conflict with the subject matter or materials discussed in the manuscript. This includes employment, consultancies, honoraria, stock ownership or options, expert testimony, grants or patents received or pending or royalties.

No writing assistance was utilized in the production of this manuscript. 


\section{References}

1 Nguyen EV, Gharib SA, Schnapp LM et al. Shotgun MS proteomic analysis of bronchoalveolar lavage fluid in normal subjects. Proteomics Clin. Appl. 8, 737-747 (2014).

2 Kingma PS, Whitsett JA. In defense of the lung: surfactant protein A and surfactant protein D. Curr. Opin. Pharmacol. 6, 277-283 (2006).

3 Serrano AG, Pérez-Gil J. Protein-lipid interactions and surface activity in the pulmonary surfactant system. Chem. Phys. Lipids 141, 105-118 (2006).

4 Kumar A, Bicer M, Pfeffer P et al. Inhaled nanoparticles develop a complex protein corona: biological importance and implications for sustained release formulations. Presented at: Drug Delivery to the Lungs - DDL25. Edinburgh, UK, 10-12 December 2014

5 Monopoli MP, Walczyck D, Campbell A et al. Physicalchemical aspects of protein corona: relevance to in vitro and in vivo biological impacts of nanoparticles. J. Am. Chem. Soc. 133, 2525-2534 (2011).

6 Tenzer S, Docter D, Kuharev J et al. Rapid formation of plasma protein corona critically affects nanoparticle pathophysiology. Nat. Nanotech. 8, 772-781 (2013).

7 Ruge CA, Schaefer UF, Herrmann J et al. The interplay of lung surfactant proteins and lipids assimilates the macrophage clearance of nanoparticles. PLoS ONE 7(7), e40775 (2012).

8 Schleh C, Rothen-Rutishauser B, Kreyling WG. The influence of pulmonary surfactant on nanoparticulate drug delivery systems. Eur. J. Pharm. Biopharm. 77, 350-352 (2011).

9 Dailey LA, Jekel N, Fink L et al. Investigation of the proinflammatory potential of biodegradable nanoparticle drug delivery systems in the lung. Toxicol. Appl. Pharmacol. 215(1), 100-108 (2006).

10 Nassimi M, Schleh C, Lauenstein $\mathrm{H}$ et al. A toxicological evaluation of inhaled solid lipid nanoparticles used as a potential drug delivery system for the lung. Eur. J. Pharm. Biopharm. 75, 107-116 (2010).
11 Harush-Frenkel O, Bivas-Benita M, Nassar T et al. A safety and tolerability study of differently-charged nanoparticles for local pulmonary drug delivery. Toxicol. Appl. Pharmacol. 246(1-2), 83-90 (2010).

12 Jones M-C, Jones SA, Riffo-Vasquez Y et al. Quantitative assessment of nanoparticle surface hydrophobicity and its influence on pulmonary biocompatibility. J. Control. Release 183(1), 94-104 (2014).

13 Dailey LA. Considerations for the design of toxicity studies of inhaled nanomedicines. In: Nanotoxicology: From In Vivo and In Vitro Models to Health Risks. Sahu SC, Casciano DA (Eds). Wiley, Chichester, England (2009).

14 Beck-Broichsitter M, Ruppert C, Schmehl T et al. Biophysical investigation of pulmonary surfactant surface properties upon contact with polymeric nanoparticles in vitro. Nanomed. Nanotechnol. Biol. Med. 7, 341-350 (2011).

15 Banquy X, Suarez F, Argaw A et al. Effect of mechanical properties of hydrogel nanoparticles on macrophage cell uptake. Soft Matter 5, 3984-3991 (2009).

16 Son Y-J, McConville JT. Development of a standardized dissolution test method for inhaled pharmaceutical formulations. Int. J. Pharm. 382, 15-22 (2009).

17 Salvati A, Pitek AS, Monopoli MP et al. Transferrinfunctionalized nanoparticles lose their targeting capabilities when a biomolecule corona adsorbs on the surface. Nat. Nanotechnol. 8, 137-143 (2013).

18 Kendall M, Ding P, Mackay RM et al. Surfactant protein D (SP-D) alters cellular uptake of particles and nanoparticles. Nanotoxicology 7, 963-973 (2013).

19 Oosting RS, Wright JR. Characterization of the surfactant protein A receptor: cell and ligand specificity. Am. J. Physiol. 267, L165-L172 (1994).

20 Caracciolo G, Cardarelli F, Pozzi D et al. Selective targeting capability acquired with a protein corona adsorbed on the surface of 1,2-dioleoyl-3-trimethylammonium propane/ DNA nanoparticles. ACS Appl. Mater. Interfaces 5, 13171-13179 (2013). 